\title{
Group Recommendation Systems Based on External Social-Trust Networks
}

\author{
Guang Fang, Lei Su $(\mathbb{D}$, Di Jiang, and Liping Wu \\ Kunming University of Science and Technology, Kunming 650051, China \\ Correspondence should be addressed to Lei Su; s28341@hotmail.com
}

Received 14 February 2018; Accepted 16 April 2018; Published 29 May 2018

Academic Editor: Huimin Lu

Copyright (C) 2018 Guang Fang et al. This is an open access article distributed under the Creative Commons Attribution License, which permits unrestricted use, distribution, and reproduction in any medium, provided the original work is properly cited.

With the development of social networks and online mobile communities, group recommendation systems support users' interaction with similar interests or purposes with others. We often provide some advices to the close friends, such as listening to favorite music and sharing favorite dishes. However, users' personalities have been ignored by the traditional group recommendation systems while the majority is satisfied. In this paper, a method of group recommendation based on external socialtrust networks is proposed, which builds a group profile by analyzing not only users' preferences, but also the social relationships between members inside and outside of the group. We employ the users' degree of disagreement to adjust group preference rating by external information of social-trust network. Moreover, having a discussion about different social network utilization ratio, we proposed a method to work for smaller group size. The experimental results show that the proposed method has consistently higher precision and leads to satisfactory recommendations for groups.

\section{Introduction}

In recent years, the research of recommendation system and mobile computing has developed very rapidly, and many kinds of recommendation systems have appeared, for example, mobile recommendation system, context-aware recommendation system, and social network recommendation system. However, most of the current system can only be worked for a single user. In fact, many daily activities are carried out by a crowd of people from different regions, such as watching movies or TV programs, going to restaurant for meal, and traveling and getting service in public. Therefore, it is necessary for the system to consider suggestion of a certain number of people, which is called GRS (group recommendation systems) [1].

In GRS, group members' preferences may be similar or different. How to get the common preference of group members, alleviate the conflict among group members and make the recommendation result as much as possible to meet the needs of all group members, which is the key problem to be addressed $[2,3]$.

The current social network group recommendation systems consider both the strength of the relationship between the members of the group into account [4-6] and the influence of social network information on each group members [7-9] and finally generate group recommenders through aggregation strategies. At present, the main influence of social network is the social impact of the group members on the group recommendation systems. At first, when the group preferences are inconsistent, those systems take care of preference of the members with more social influence and ignore some intentions of the members with small social influence. In addition, it is unreasonable that the present GRS still consider the influence of social networks while the group has reached consensus. This paper aims at correcting preference rating by social-trust networks when group rating of item cannot reach consensus. Specifically, this paper uses the real ratings of the external members trusted by the group members to correct the predicted rating of some item. When the disagreement of group is small, namely, group tendency reaches consensus, the influence would be reduced by socialtrust networks on GRS, so as to dynamically adjust the influencing factors of social networks and reduce the error on GRS.

In addition, whole group members are asked to be in the same social network on present social network 
recommendation system [4, 5, 7-9], and the members of other social networks could be ignored. The method proposed in this paper is that some users who dislike communicating with others on social network and are not in the same social network can benefit from this as well.

The structure of this paper is as follows. In Section 2, we introduce the related work recommended by the social network, and Section 3 elaborates the method based on external trust social network proposed in this paper. Section 4 introduces the experimental results. Section 5 summarizes the full text and discusses future work.

\section{Related Work}

2.1. Group Recommendation Systems. GRS [3] usually generate group preferences through aggregating individual's ratings. According to the paper by Jameson and Smyth [1], the main approaches to generate the preference aggregation are merging the recommendations made for individuals, aggregation of ratings for individuals, and constructing a group preference model. Supan et al. [10] present a compilation of the most important preference aggregation techniques. These basic approaches merge the ratings predicted individually for each item to calculate a global prediction for the group. The selection of a proper aggregation strategy is a key element in the success of recommendation. The work by Ricci et al. [11] describes a series of experiments that were conducted with real users in order to determine which strategy performs best. These experiments show that the average and the average without misery strategies perform best from the users' point of view because they seem to obtain similar recommendations to those that emerge from an actual discussion in a group of "humans".

Group recommendation systems could be classified into two main categories $[12,13]$ : those which perform an aggregation of individuals' preferences to obtain a possible group evaluation for each candidate item and those which perform an aggregation of individuals' models into a single group model and generate suggestions based on this model. In the first method, an individual-based recommendation system is first used to generate recommendations for each group member, then a group consensus function is used to merge the individual recommendations and select ones that are most suitable for the whole group. In the second method, a pseudo user profile is generated from all group members, and an individual-based recommendation system is then used at run time to generate recommendations for the pseudo user. By considering the recommendations for individual group members and merging [7] them at run time to generate group recommenders, this GRS architecture can easily accommodate dynamic groups and tailor its recommendations for each specific scenario.

The individual recommender implements the Collaborative Filtering algorithm described in Kelleher and Bridge [14] and group recommendation has largely been studied in the context of Collaborative Filtering (CF) $[8,15,16]$. We have chosen this algorithm because it is widely used to recommend items when the modeling of user preferences is not a valid option (as in most of real scenarios [17-19] and others
[20-23]). This algorithm requires users to rate an initial set of items. Then, those ratings are used to estimate the predicted rating for an unrated item.

Some instances have been applied on GRS, such as MusicFX [24] which obtains the information about the clients' interests from a database that stores their music genre preferences (previously and explicitly specified). Some systems make the suggestions which would not be particularly abrupt and maintain the consistency of the recommended style. In order to avoid abrupt music recommendation, music to be played would be considered in FlyTrap [25]. Another system that takes previous selections into account is PoolCasting [26]. It uses a Case-Based Reasoning system to generate a sequence of songs customized for a mobile community of listeners. To select each song in the sequence, first a subset of songs musically associated with the last song of the sequence is retrieved from a music pool; then the preferences of the audience expressed as cases are reused to customize the selection for the group of listeners; finally, listeners can revise their satisfaction (or lack of) for the songs they have heard. Let's Browse [27] first creates the individual profiles as a set of about 50 keyword-weighted pairs obtained by a crawler from the user's page. Then the group preference model results from a simple linear combination of the individuals' profiles.

In addition to personal information, there are some other pieces of information which would be taken into account [28, 29], and in another interesting content-based recommendation system there is Pocket Restaurant Finder [30], which recommends restaurants for groups of people based on user location and the culinary characteristics of the restaurant. McCarthy proposes the Pocket Restaurant Finder that recommends restaurants to groups of people considering their culinary preferences and location. Specifically, the recommender uses information like travel distance, expected facilities, cuisine desired, and budget planned. When using the recommendation system, the group members have to express explicitly and the desired values for the four features individually, and they also need to order the features in a level of priority. Then, Pocket Restaurant Finder computes the recommendation by applying an average preferences aggregation method. The restaurants are displayed in a ranked list that matches the group's likes.

\subsection{Social Network Recommendation System. One of the} important influencing factors of the GRS is the social relationship among group $[8,9,31,32]$. Research shows that users prefer to accept the recommendation of trusted users rather than anonymous user [5].

Gartrell et al. [7] proposed that the social network merges into the group recommendation systems for the first time. The method is called Rule-Based Group Consensus Framework, which not only considers the group members' interest, but also describes the group members' weight difference. The system takes social relations, social frequency, and professional level into account.

With the development of the Internet, social platform has been focused by researchers as well. HappyMovie [8] is an online GRS on Facebook; moreover researchers need 
to handle two tests: one is personality test, getting users' personality through TKI [33], and personality is divided from selfishness to tolerance, a total of five levels. Usually selfish users are not affected by others and tolerant users are easily impacted, in order to reach consensus in group, making vulnerable users change their recommendations. The other one is building user preference model through choosing enjoyed movies by themselves. Then group suggestion is generated through aggregating individual preferences.

SocialGR [9] is also a real research system, the system takes many aspects of social factors into account, and the main consideration is Trust Relationship (TR), Social Similarity (SS), and Social Centrality (SC). TR reflects the cohesion between two members by analyzing their affective relation. SS reflects the likeness between members, that is, shared activities, likes, friends, or interests. SC reflects members' reputations in the social network. The basic recommendation system utilizes a Collaborative Filtering recommendation system. And group recommender is determined through the Maximizing Average Satisfaction (MAS) strategy.

Current social networks and group recommenders usually considered the strength of the relationship among the members of the group; the tie among members would affect the results of GRS. Though some papers [4-7, 9] considered the social factors such as personality, trust, personal expertise, and social status of the members; once the information was acquired, the steps of the social networking group recommendation systems were as follows: At first, fetching the list of preference of all group members by individual recommendation system; then, increasing or decreasing the weight of a group of members of the article via social factors; finally, getting the ultimate results of group recommendation systems by some aggregation strategies, which consider the individual recommendation and weight. These methods utilize the social factors more or less; group members' weight is considered in ones; it means that a few of members would be ignored because of some group members play a decisive role, leading to the tendency to influence influential members. Therefore, this paper focuses on the impact of external members on the GRS, the information of external members, and internal members is taken as a whole, respectively.

\section{External-Based Social-Trust Networks Group Recommendation Systems}

3.1. GRS Calculation Framework. As to previous group recommendations in social networks, they consider the strength of the relationship between group members $[4,5]$ and the impact of social network information on each group member [7-9], hence generating group recommendation by the aggregation strategy. At present, the main impact of the social network on the group recommendation systems is the social influence of the group members. On the one hand, when the group preferences are inconsistent, those systems take care of preference of the members with more social influence and take no notice of some intentions of the members with small social influence; on the other hand, it is unreasonable that those system still consider the influence of social networks while the group has reached consensus. The idea of this paper includes the following: correcting preference rating through social-trust networks when group rating of item cannot reach consensus. Specifically, this paper uses the real ratings of external members that are trusted by the group members to correct the predicted rating of items. When the disagreement of group is small, namely, group tendency reaches consensus, the influence would be reduced on GRS by social network, so as to dynamically adjust the influence of social networks and reduce the error on GRS. In fact, most people hope to persuade others to follow their advices when faced with the conflict and large disagreement among the group. At that moment, if there is a role, which you trusted and could tell you what is the best choice, perhaps you would change your mind. Watching a movie with your friends, for instance, you may not intend to do it with them but someone you trusted would change your mind in that just he watched it before and has felt well. This scenario shows correcting group preference by new information when degree of group disagreement is large in GRS, and the group is able to reach consensus.

At first, fetching the list of preference of each group member by Collaborative Filtering, and ensuring a special group (by randomly selected group or fasten group), and getting the results of group recommendation systems by some aggregation strategies, the above progress is called classical group prediction. Then, calculating disagreement of item $i$ by predictions of group members, disagreement is greatly affected by external trust networks and vice versa. The frame and formula of our method are Figure 1 and formula (1).

$$
\operatorname{gpred}(G, i)= \begin{cases}\left(1-\lambda_{i}\right) \cdot \operatorname{CGP}(G, i)+\lambda_{i} \cdot \mathrm{OAR}(\mathrm{OG}, i) & \\ \operatorname{CGP}(G, i), & \text { if } \mathrm{OAR}(\mathrm{OG}, i)=\varnothing \\ \operatorname{OAR}(\mathrm{OG}, i), & \text { if } \mathrm{CGP}(G, i)=\varnothing\end{cases}
$$

Here, $G$ refer to a group and OG (out of group) represents information of external group, namely, users that group members trusted. $\operatorname{CGP}(G, i)$ (classical group prediction) is predictions of item $i$ by individual recommendation system and some aggregation strategies. OAR(OG,i) (outer actual rating) represents actual ratings of external members of group and $\lambda_{i}$ is a dynamic balance factor of external members and internal members of item $i$, balancing ratings of 

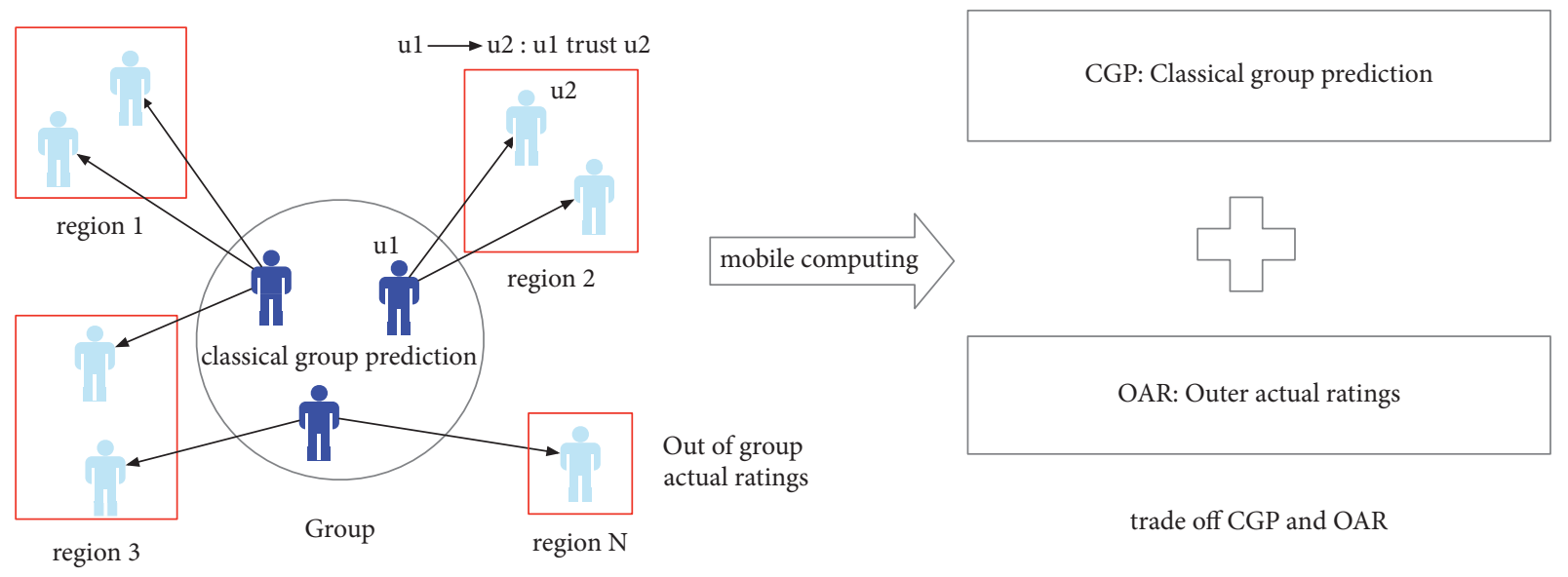

FIGURE 1: Frame of group recommendation systems.

recommendation between external and internal members. Џ represents a aggregation strategy. Note that, if OAR $(\mathrm{OG}, i)=$ $\varnothing$, then there are no real ratings of item $i$ by trusted users, and results calculated by $\operatorname{CGP}(G, i)$ would be selected. Else if $\operatorname{CGP}(G, i)=\varnothing$, then there are no predicted ratings of item $i$ by group users, and results calculated by $\mathrm{OAR}(\mathrm{OG}, i)$ would be utilized.

We have adjusted the intensity of the external influence dynamically. When the disagreement of group members is large, we need the external real score to correct. The bigger the disagreement is, the greater the external influence of group members' external influence would be. When the disagreement of group members is small, the opinions of the group members need to be retained. The smaller the disagreement is, the less the group members would be influenced externally. For example, when two people decide to watch a movie together, even if someone recommends a good movie, it may not be easy to influence the decision of the two. We need to solve two problems: one is how to determine the value of $\lambda$, and the other is how to solve $\operatorname{OAR}(\mathrm{OG}, i) \operatorname{CGP}(G, i)$ (classical group prediction) represents predictions of item $i$ by individual recommendation system and some aggregation strategies; the formula is as follows:

$$
\operatorname{CGP}(G, i)=\coprod_{\forall u \in G} \operatorname{pred}(u, i)
$$

$\coprod$ represents a aggregation strategy and $\operatorname{pred}(u, i)$ represents user $u$ 's predicted rating on item $i$ by individual recommendation system.

In $\mathrm{OAR}(\mathrm{OG}, i)$, the actual ratings of external members, which are relative to group members, would be introduced into GRS by social network. Moreover, the object of each user trust may be more than one, and two points need to be considered on external actual information: degree of trust and obtaining actual rating set of members that are trusted; the formula is as follows:

$$
\operatorname{OAR}(\mathrm{OG}, i)=\coprod_{\forall u \in G, v \in \mathrm{OG}} t_{u v} \cdot \operatorname{actual} \_ \text {rating }(v, i) .
$$

Here, $G$ refers to a group, OG (out of group) represents information of external group, namely, users that group members trusted, $\bigsqcup$ represents a aggregation strategy, $t_{u v}$ represents degree of trust of user $u$ on user $v, t_{u v} \in[0,1]$, 0 is trust scarcely, and 1 refers to trust completely. The more the influence, the high the degree of trust in external actual information; actual_rating $(v, i)$ represents actual rating of user $v$ on item $i$.

\subsection{Dynamic Adjustment of Parameter $\lambda$}

3.2.1. LVD. This approach utilizes disagreement and refines a little on disagreement. Firstly, calculate the unbiased estimate of predicted ratings of group members, namely, sample variance in order to compute the number of points far away from the center in group preference set. The method is called LVD (lambda via disagreement), and the formula is as follows:

$$
\begin{aligned}
\operatorname{dis} & (G, i) \\
= & \frac{1}{|G|-1} \sum_{u \in G}\left[\operatorname{pred}(u, i)-\frac{\sum_{v \in G} \operatorname{pred}(v, i)}{|G|}\right]^{2}
\end{aligned}
$$

and the calculating formula of $\lambda$ is as follows:

$$
\begin{aligned}
\lambda= & \frac{1}{|G|} \sum_{u \in G} \mathbb{1}\left[\left(\operatorname{pred}(u, i)-\frac{\sum_{v \in G} \operatorname{pred}(v, i)}{|G|}\right)^{2}\right. \\
& >\operatorname{dis}(G, i)] .
\end{aligned}
$$

Here, $|G|$ refers to the number of a group and indicator function is introduced: $\mathbb{1}[x]$ refers to that if $x$ is true, then expression is 1 , unless 0 . $\operatorname{pred}(u, i)$ represents user $u$ 's predicted rating on item $i$ by individual recommendation system.

3.2.2. LVTP. Owing to the structural of LVD is simple, some requirements should be met on the size and predicted distribution of groups. On the one hand, in the case with which 
TABLE 1: $\lambda$ via disagreement.

\begin{tabular}{lcccc}
\hline & User1 & User2 & dis & $\lambda$ \\
\hline Item $_{A}$ & 0 & 4 & 8 & 0 \\
Item $_{B}$ & 0 & 5 & 12.5 & 0 \\
\hline
\end{tabular}

TABLE 2: $\lambda$ via disagreement.

\begin{tabular}{lccccc}
\hline & User1 & User2 & User3 & dis & $\lambda$ \\
\hline Item $_{A}$ & 0 & 1 & 4 & 4.33 & 0.33 \\
Item $_{B}$ & 0 & 1 & 5 & 7.0 & 0.33 \\
\hline
\end{tabular}

only two members cannot cope, Table 1, the disagreements (dis) are 8 and 12.5, respectively, and the values of lambda are both 0 , which means that adjustment is unnecessary on recommendations of external social network, and it is unreasonable. On the other hand, Table 2, the disagreements (dis) are 4.33 and 7.0, respectively, and the values of lambda are both 0.33 ; something looks well but still cannot reflect well with big disagreement on social network. Both of the above two cases with the large disagreement are observed, however having small values of lambda; as a result, this method cannot deal with the situation of mere few people.

In order to address the above issue, the method LVTP (lambda via two parts) is proposed in that case as the following steps: firstly, the standard value $\alpha_{r i}$, a balancing item $i$, would be set; secondly, utilizing standard value $\alpha_{r i}$ divides the predictions of group members into different two parts, a big one is Greater $r i$ and a small one is Les $r i$; finally, calculate lambda value on each item $i$. The formula is as follows:

$$
\lambda_{i}=\frac{\mathbb{1}\left[\text { Greater }_{r i} \wedge \text { Les }_{r i}>\varnothing\right]\left(\overline{\text { Greater }_{r i}}-\overline{\text { Les }_{r i}}\right)}{\gamma+\max \left(\text { Greater }_{r i}\right)} .
$$

Here $\overline{\text { Greater }_{r i}}$ represents the average value of bigger than standard value in rating set. Indicator function is introduced: $\mathbb{1}[x]$ refers to that if $x$ is true, then expression is 1 , unless 0 . Note that, in step 3, if Greater $r i=\varnothing$ or Les $r i=\varnothing$, which means that ratings of group on item $i$ reach consensus and indicates that group members are either like or dislike item $i$, then $\lambda_{i}=0$, which means that social network has almost no influence on item $i$. Particularly, $\gamma$ is a smooth factor, in order to avoid if $\left(\overline{\text { Greater }_{r i}}-\overline{\text { Les }_{r i}}\right)=\max \left(\right.$ Greater $\left._{r i}\right)$ result in the GRS neglect of group's suggestions completely, as well as unreasonable.

With regard to the choice of standard values, this paper considers the following three aspects: (1) The mid value of the range can be evaluated; for example, the maximum score for a certain item is 10 and the lowest is 1 . The mid value is $(10+$ $1) / 2=5.5$, and 5.5 is the standard value. (2) The mean of all predictions of item $i$ in the training data is the standard value, and the standard values for each item are different. (3) The median of all predictions of item $i$ in the training data is the standard value, and the standard values for each item are different.
TABLE 3: Average strategy.

\begin{tabular}{lcccc}
\hline & User1 & User2 & User3 & gpred \\
\hline Item $_{A}$ & 5 & 1 & 3 & 3 \\
Item $_{B}$ & 4 & 2 & 3 & 3 \\
\hline
\end{tabular}

TABLE 4: Maximum satisfaction.

\begin{tabular}{lcccc}
\hline & User1 & User2 & User3 & gpred \\
\hline Item $_{A}$ & 5 & 1 & 3 & 5 \\
Item $_{B}$ & 4 & 2 & 3 & 4 \\
\hline
\end{tabular}

TABLE 5: Calculate disagreement.

\begin{tabular}{lcccc}
\hline & User1 & User2 & User3 & dis \\
\hline Item $_{A}$ & 5 & 1 & 3 & $4 / 3$ \\
Item $_{B}$ & 4 & 2 & 3 & $4 / 3$ \\
\hline
\end{tabular}

\subsection{Group Recommendation Systems Based on \\ External Social-Trust Networks}

3.3.1. A Description of the Aggregation Strategy. In group recommendation systems, preference fusion refers to integrating the preferences of group members and preference fusion is also known as the aggregation strategy [11] or the Aggregate Rules [34]; for the unity of terminology, this paper uses the term aggregation strategy. Ricci et al. [11] described 10 aggregation strategies in detail, and having relatively better strategies such as average strategy and average without misery in a series of experiments of another paper.

In average strategy, the group rating for a particular item is computed as the average rating over all individuals.

$$
\operatorname{gpred}(G, i)=\frac{1}{|G|} \sum_{u \in G} \operatorname{pred}(u, i)
$$

where $|G|$ is the group size and $\operatorname{pred}(u, i)$ is the predicted rating for each user $u$ and every item $i$. Table 3 shows example of average strategy, and gpred is predicted rating of group.

Maximum satisfaction strategy refers to the greatest rating item in whole group, ignoring the others' lower ratings.

$$
\operatorname{gpred}(G, i)=\operatorname{maxpred}(u, i), \quad u \in G,
$$

where maxpred refers to max prediction rating in set. Table 4 shows the example about the maximum satisfaction.

Group disagreement with the project $[1] \operatorname{dis}(G, i)$ indicates the degree of difference of users in the group $G$ on the predicted score of the project $i$.

$$
\operatorname{dis}(G, i)=\frac{1}{|G|} \sum_{u \in G}[\operatorname{pred}(u, i)-\operatorname{mean}(G, i)]^{2} \text {. }
$$

mean $(G, i)$ denotes the mean of the group prediction ratings of item $i$. Table 5 gives an example of the bifurcation calculation.

3.3.2. GRITrust Algorithm. According to the description of $\lambda$ and $\mathrm{OAR}(\mathrm{OG}, i)$ on Sections 3.1 and 3.2, the result can 


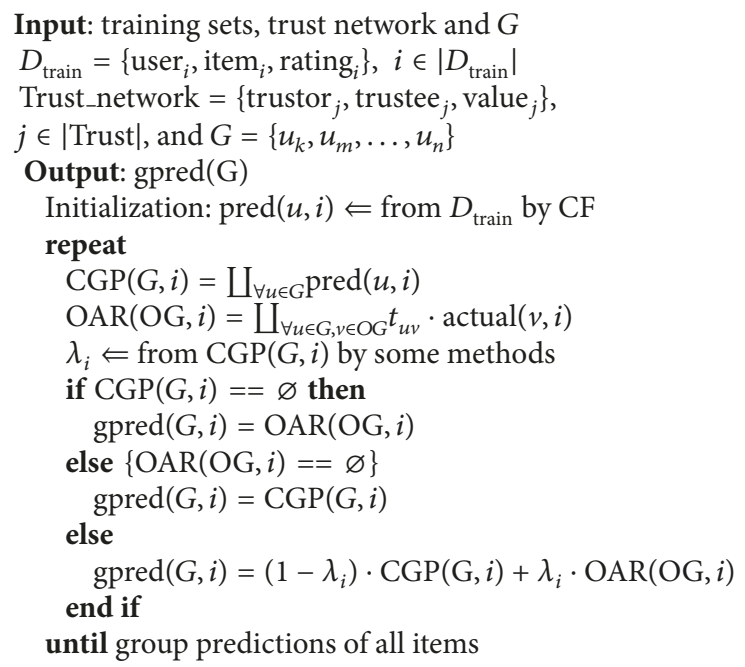

Algorithm 1: GRITrust.

TABle 6: Actions.

\begin{tabular}{lcc}
\hline \# users & \# items & \# ratings \\
\hline 1,508 & 2,071 & 35,497 \\
\hline
\end{tabular}

TABle 7: Trust relationships.

\begin{tabular}{lcc}
\hline trustor & trustee & trust_value \\
\hline 2 & 966 & 1 \\
\hline
\end{tabular}

be computed with those parameters. Note that, if there is no prediction on $\operatorname{CGP}(G, i)$ but on $\operatorname{OAR}(\mathrm{OG}, i)$. The group recommendation systems' result is not empty but $\mathrm{OAR}(\mathrm{OG}, i)$. Above method can ease the cold start problem on GRS. Sometimes, the user has no any ratings, and the system can able to help him to find some better answers.

In this paper, the algorithm named group recommender involve trust (GRITrust) network pseudocode is in Algorithm 1 .

\section{Experiments}

4.1. Experimental Data. In order to verify proposed method all performance experiments were conducted on an open data.

Dataset. We have used the FilmTrust (https://www.librec.net/ datasets/filmtrust.zip) [35] ratings dataset for evaluation purposes. The statistics of this dataset are shown in Tables 6 and 7 . And rating range is $[0.5,4]$.

The dataset has been built as follows: firstly, the actual rating of users who have evaluated movies has been divided into 8 to 2, which means the preferences of item for each people; for instance, some users have 10 ratings of different items, training set has 8 ratings, and test set has 2 . However some few users have no more than 5 ratings and cannot be split, and we will drop it. Then the training set has 1482 users and test set has 1421 ones, because a bit of users have no rating counts enough. Particularly, only there are 609 users who can trust one or more other ones which means just 41 percent of users would use social network in the training set. Let $p$ be ratio value that is numbers of users on the network to training set population, and the value is 0.41 . We can calculate the probability of using social networks as $1-(1-p)^{n}$ via Binomial Distribution when group size is $n$. And if we can get $n=2,3,4$ then the probability is $0.65,0.80,0.87$. Obviously, the value is lower than others when $n$ equals 2 . Furthermore, in order to verify the reliability of the proposed method, we would separately discuss the case where the group size is 2.

In this paper, a randomized grouping method is used to conduct the experiment. In the group selection, we noticed that if we experiment with a random group of three people, considering each possibility that brings a large computing cost, for example, when there are 1000 users in dataset, and there are 166, 167,000 randomly selected combinations of 3 people. Obviously, if the numbers and the sizes of groups are appropriately increased, it is impossible to calculate them one by one. For this sake of convenience, we conducted a random sample of studies.

4.2. Evaluation Method Description. In order to evaluate the effectiveness of the proposed method, the root mean square error (RMSE) [7] is used as evaluation method which can assess the quality of the recommended system in terms of accuracy, and the formula is as follows:

$$
\operatorname{RMSE}=\sqrt{\frac{\sum_{i=1}^{N}(\operatorname{gpred}(G, i)-\operatorname{actual}(G, i))^{2}}{N}},
$$

where $N$ is the number of items recommended in the group. 
TABLE 8: Explanation.

\begin{tabular}{lc}
\hline name & explanation \\
\hline baseline & without network \\
GRITrust_dis & $\lambda_{\text {LVD }}$ \\
GRITrust_mean & $\lambda_{\text {LVTP }} \alpha_{\text {mean }}$ \\
GRITrust_mid & $\lambda_{\text {LVTP }} \alpha_{\text {mid }}$ \\
GRITrust_median & $\lambda_{\text {LVTP }} \alpha_{\text {median }}$ \\
\hline
\end{tabular}

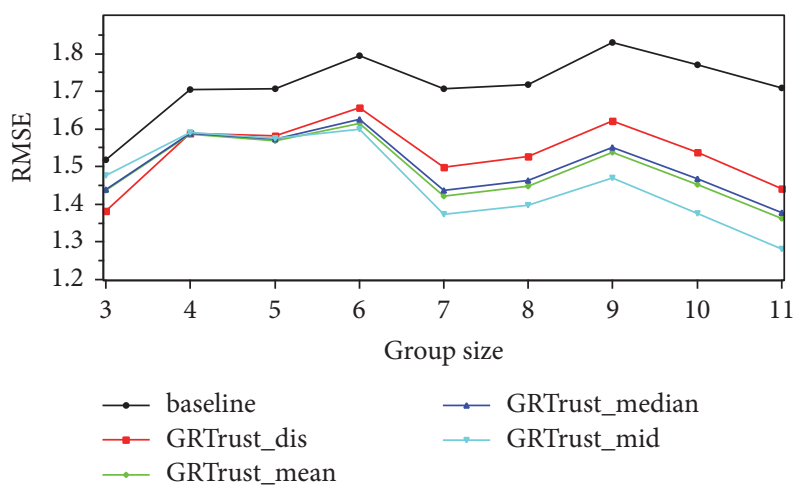

FIGURE 2: RMSE comparison of different group size by average strategy.

4.3. Randomly Divided into Groups Experiment. The basic idea of the experiment is Collaborative Filtering (CF) algorithm in the training set; each user has not seen item prediction rating, resulting in personalized recommendations. Then randomly select a group and calculate the dynamic adjustment parameters $\lambda$, finding the group members trusted in the trust network object, selecting the appropriate aggregation strategy and calculating prediction ratings of item by formula (1). Finally, comparing predictions with the test set, note that the test set also uses the same aggregation strategy.

$\lambda_{\mathrm{LVD}}$ in Table 8 indicates the method of calculating $\lambda$ by LVD and $\lambda_{\text {LVTP }}$ denotes the method of calculating $\lambda$ by LVTP. Methods of calculating standard value $\alpha$ are $\alpha_{\text {mean }}$ standard value using the true value of the sample; $\alpha_{\text {mid }}$ standard value using the middle of the range which can be evaluated; $\alpha_{\text {median }}$ standard value using the median of the real sample.

According to group size we have randomly selected 100 times in this experiment, and group size is from 3 to 11. Figure 2 illustrates the performance of baseline, GRITrust_dis, GRITrust_mean, GRITrust_mid, and GRITrust_median with different group size. When group size is 3, GRITrust_dis method is the best than others; it that means that LVP is better than LVTP. In addition, if group size is greater than 5, then LVTP is better than LVP; especially, GRITrust_mid is better than others.

In order to further verify the effectiveness of the proposed method, we carried out the same experiment on the maximum satisfaction strategy. The results of experiment are shown in Figure 3. The GRITrust_dis method is also significantly better than the other methods when group size is 3 , but when group size is greater than 5 , the error can still be smaller than baseline, and LVTP can get lower RMSE.

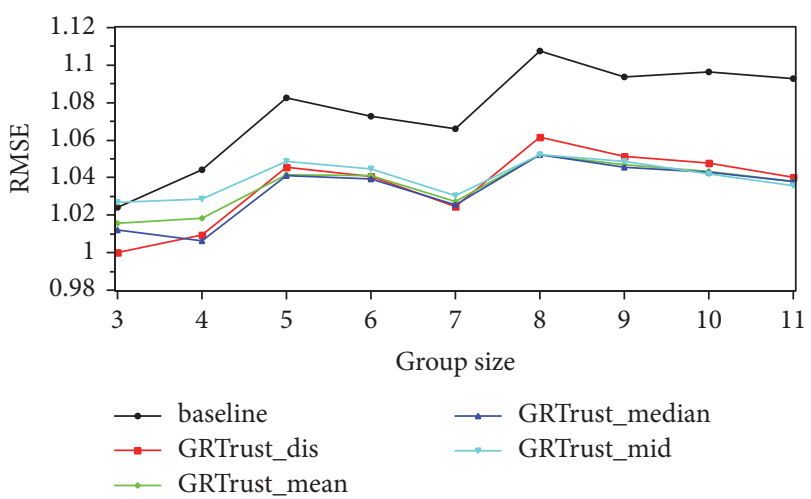

FIGURE 3: RMSE comparison of different group size by maximum satisfaction strategy.

Through the above two experiments, we show the effectiveness of the proposed method in group recommendation systems, when the large group disagreement is increasing the influence of the social network and vice versa.

4.4. Social Networking Utilization Ratio in Group Recommendation. According to the research, there are three characteristics in group recommendation systems with social network: (1) not all users in social networks, which means that some users do not use social networks; (2) in the current social network, some users do not pay attention to others; (3) some users have little or no rating information on the item. Therefore, we can not guarantee getting the useful information at every time when visiting social networks. The definition of social network utilization is given below.

Definition 1. In group recommendation systems with social network, $m$ is group recommendations, $n$ is social networks visited and used through recommendation system, and we define the ratio of $n$ and $m$ as the social network utilization ratio $r_{\text {socail }}$.

$$
r_{\text {socail }}=\frac{n}{m}, \quad r_{\text {socail }} \in[0,1] .
$$

In order to verify the proposed method that is positively correlated with the social network utilization in group recommendation systems with social network, we used the same dataset and method as the random sample experiment except that different utilization ratios of social network were chosen to study the experiment. Among them, $r_{\text {socail }}=\{0.2,0.4,0.6,0.8,1.0\}$, and the group size is from 3 to 10; for example, G3 indicates that the group size is 3.

In Figure 4, using average strategy, we compared RMSE and social network utilization ratio. The conclusion can be reached that the decline of the Root Mean Square Error has little relationship with the size of the group; however, it is positively correlated with the social network utilization. Figure 5 shows the descent percent by social network utilization ratio. In Figure 6, we evaluated RMSE of $20 \%, 40 \%, 60 \%$, $80 \%$, and $100 \%$ of social network utilization ratio in different 

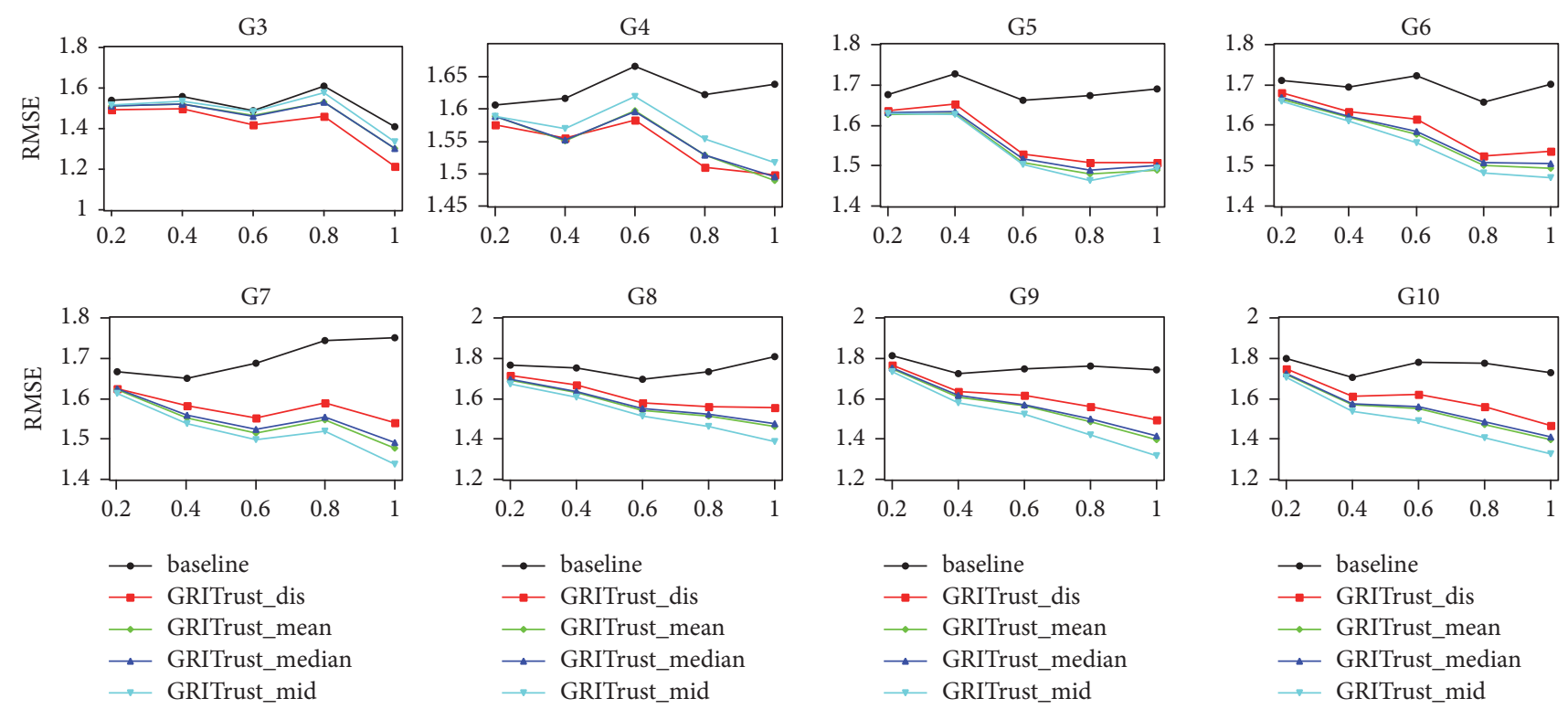

FIGURE 4: RMSE comparison of different group size and different social network utilization ratio.

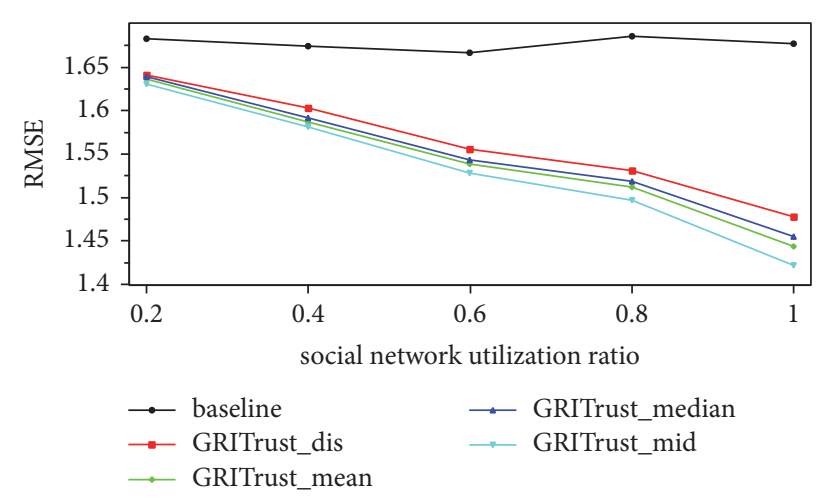

FIGURE 5: RMSE with social network utilization ratio.

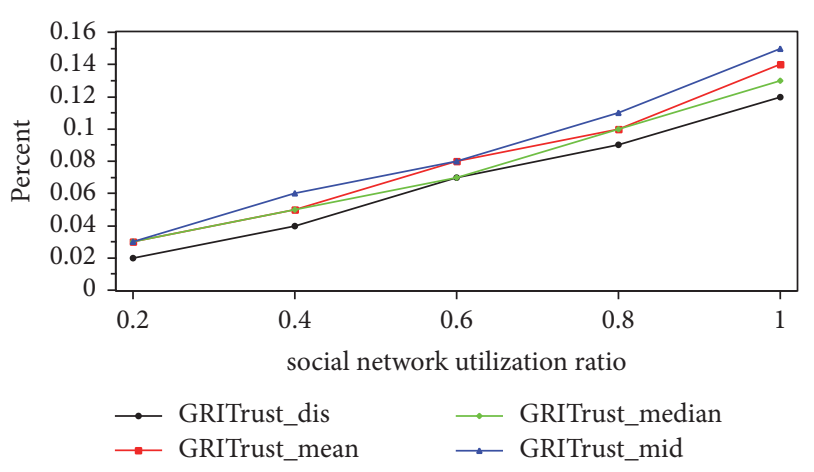

FIGURE 6: Descent percentage as social network utilization ratio.

group size. The experiment shows that RMSE decreases as the social network utilization ratio increases, and the effect achieved by GRITrust_mid is relatively good, about $4 \%$ to $16 \%$.

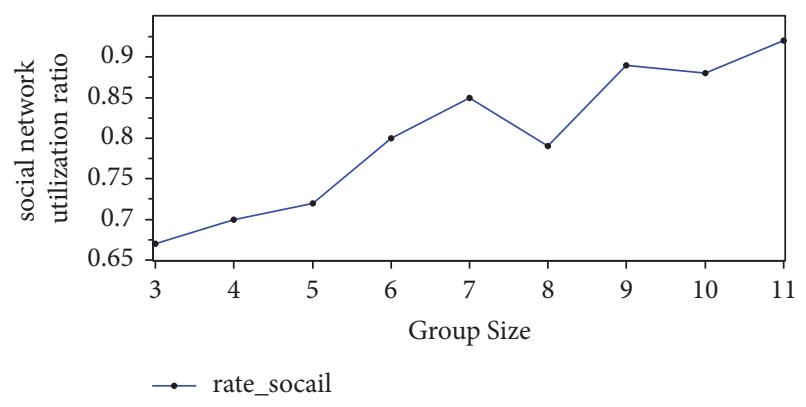

FIGURE 7: Under random sample.

Figure 7 is the social network utilization in the case of random sampling by different group size; we turn out that the larger the group is, the higher the social network utilization ratio is, and the range is $67 \%-92 \%$ in this dataset. According to Figure 6, RMSE of our proposed method decreases by about $9 \%-15 \%$.

The above experiment gives the result of using average strategy. In addition, RMSE is reduced as well by maximum satisfaction strategy with different group size in Figure 8. The result of experiment demonstrates the potential of the proposed approach.

Similarly, Figure 9 shows that the RMSE of the proposed method decreases as the social network utilization ratios increase. And GRITrust_median obtained the effect which is relatively good.

In Figure 10 we see that using method $\lambda_{\text {LVTP }}$ is better on average than method $\lambda_{\mathrm{LVD}}$ with different coverage ratios. Among them, the effect of $\alpha_{\text {median }}$ in method $\lambda_{\text {LVTP }}$ is the best, and the average drop of root mean square error is the highest, $4.4 \%$.

Through the above experimental verification, in the case of different social network utilization ratio, the higher the 

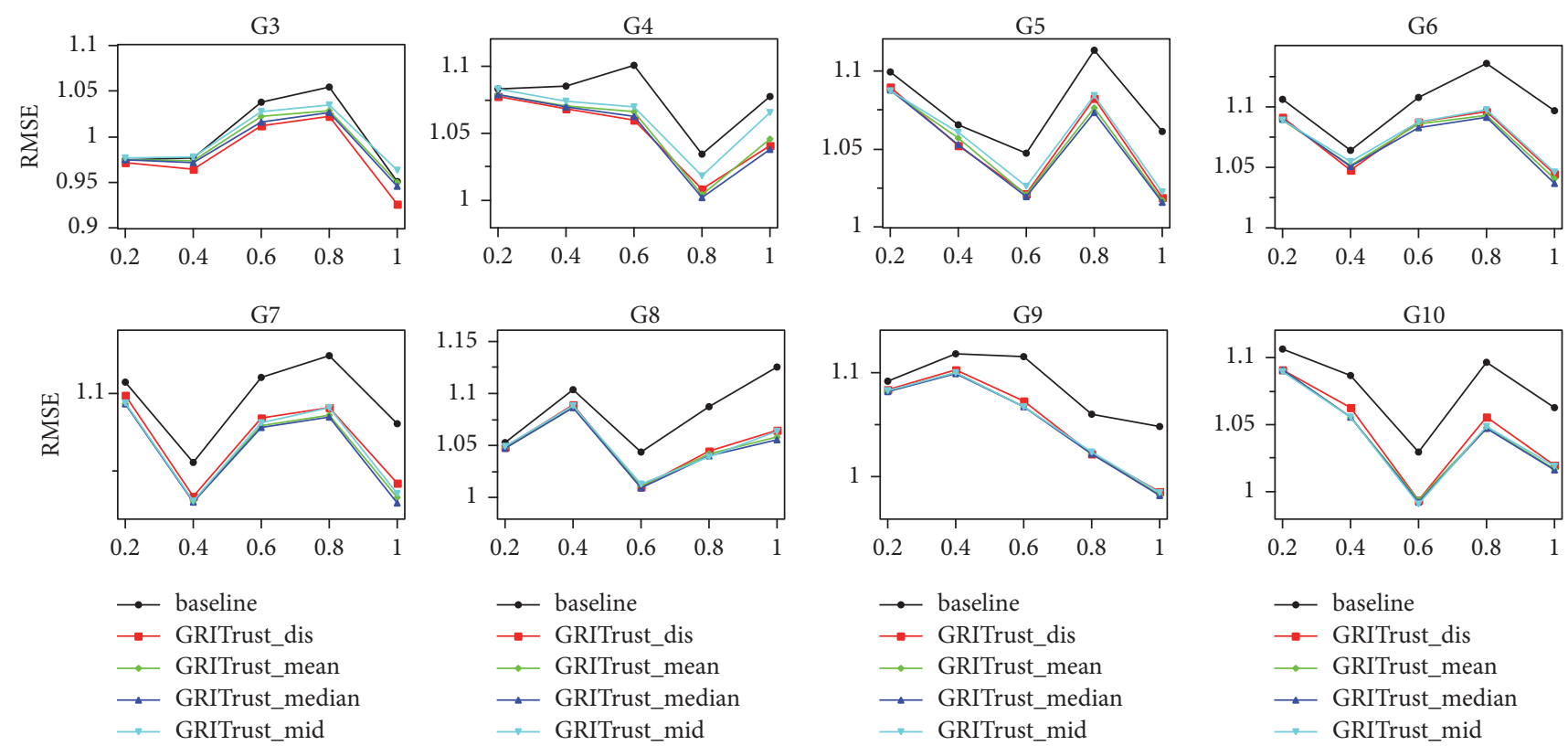

FIGURE 8: RMSE comparison of different group size and different social network utilization ratio.

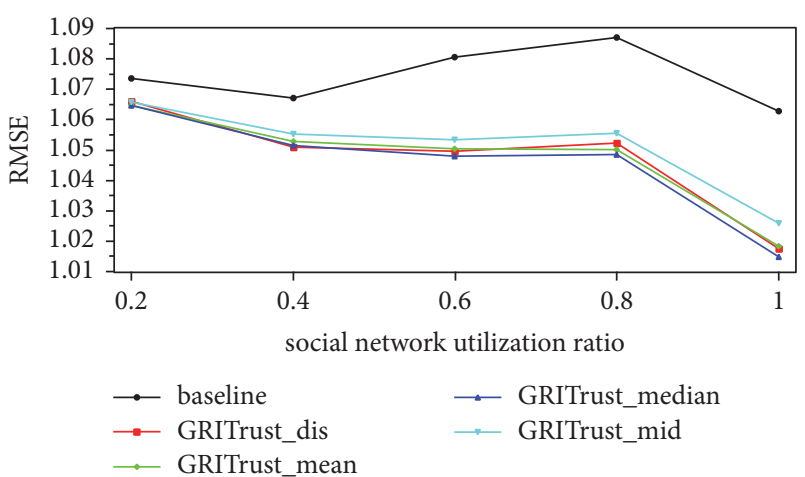

FIGURE 9: RMSE with utilization of social network.

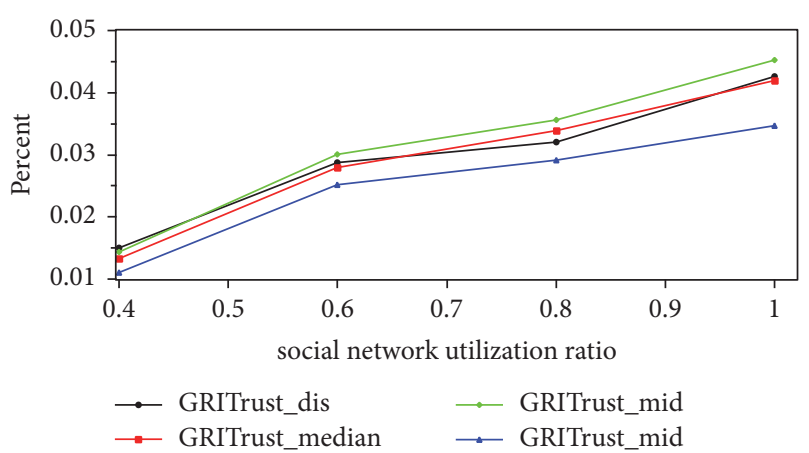

FIGURE 10: Descent percentage as social network utilization ratio.

utilization rate of social network is, the better the effect of the method proposed in this article is, although the above experiment mainly discusses the case of 3-10 users, ignoring case of group size 2 .
TABLE 9: Social network utilization ratio.

\begin{tabular}{lccc}
\hline sample size & 100 & 1000 & 10000 \\
\hline ratio & 0.48 & 0.518 & 0.5265 \\
\hline
\end{tabular}

4.5. Experiment with Group Size 2. As mentioned earlier, when the number of users in a group is 2 , the social network utilization ratio is 0.65 . In fact, we can not achieve this value in a limited sample. On the other hand, according to the three aspects mentioned in Section 4.4, it indicates that the social network utilization ratio would not be too high. As a result we conducted a random sampling experiment, the experimental results shown in Table 9; therefore, we specially discussed group size 2.

In this experiment, the group size is 2 , and the random sample times are 1000, because the method $\lambda_{\mathrm{LVD}}$ in the group size 2 according to Table 9 shows that we can not calculate the better results, so this experiment would not be considered. The experimental results are opposite in Figures 11 and 5. In the case of 3-10 users, the results are better than the other methods among the methods by $\lambda_{\text {LVTP }}$ and standard value is GRITrust_mid; however, RMSE of this result is higher than baseline as Figure 11 shows which indicates that the effect was not good. While the standard value is GRITrust_median, the RMSE is relatively low, and the effect is better than other standard values.

Similarly in the maximum satisfaction strategy experiment, Figure 12 can get the same conclusion.

According to the above two comparative experiments, when the group size is 2 , the method $\lambda_{\text {LVTP }}$ is adopted, and the root mean square error of taking $\alpha_{\text {median }}$ is the standard value that is the lowest, and the effect is the best. 


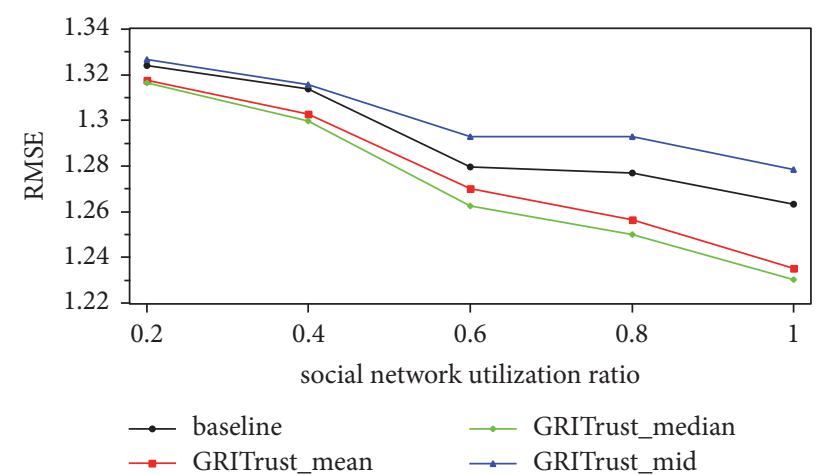

FIGURE 11: The change curve of RMSE with the social network utilization ratio, average strategy.

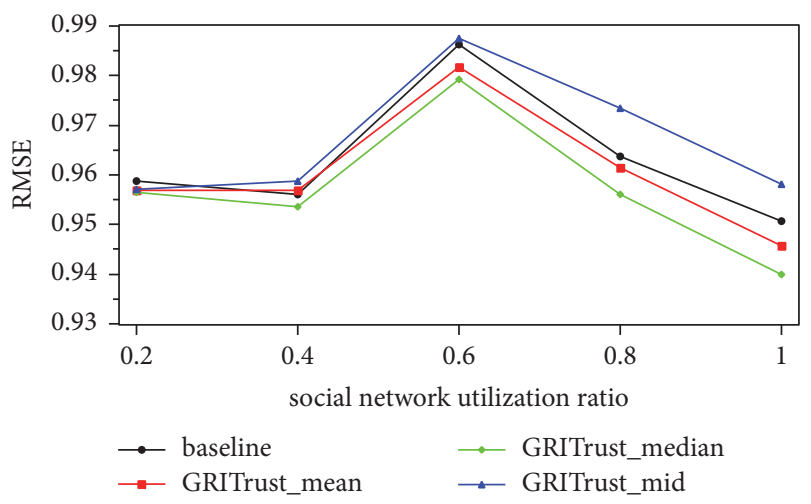

FIGURE 12: The change curve of RMSE with the social network utilization ratio, maximum satisfaction strategy.

\section{Conclusion}

This paper introduces the influence of trust network on the group recommendation system. In the traditional group recommendation, when the group preference diverges, the potential intentions of some group members will be ignored. In this paper, we use the trust network relationship in social networks to introduce group members' external real information, through a true evaluation of an item, to amend the group of a forecast of an item, when the group disagreement is small, that is, within the group to achieve the same case, to reduce the social network recommended to the group impact. Thereby dynamically adjusting the impact of social networking factors improves the quality of group recommendations. Through experiments, different aggregation strategies are used to verify the effectiveness of the proposed method in different group sizes. The error of the proposed method does not increase with the increase of the group and will remain at a relatively low level. Based on this, we further discuss the influence of social network utilization on the results of the group recommendation system. Our method shows that, in the group recommendation system, under the same group size, the utilization rate of social network is higher and the root mean square error is lower. In other words, the higher social network utilization ratio is, the better group recommendation is obtained. This shows that, for a new user, as long as the user chooses a few trusted objects, our method can get a good result.

Although some results have been achieved, it does not fully utilize all the valid information in social networks, such as user similarity, user personality, and social status. This paper aims to verify the impact of group members outside other than discussing the relationship between group members, which will be the future need to be discussed. In addition, other domains need to be considered as well except movie; the size of the data is also the direction of efforts.

\section{Data Availability}

We have used the FilmTrust ratings dataset at http://www librec.net/datasets/filmtrust.zip for evaluation purposes. The statistics of this dataset is shown in Tables 6 and 7, and rating range is $[0.5,4]$.

\section{Conflicts of Interest}

The authors declare that they have no conflicts of interest.

\section{Acknowledgments}

This work is supported by the National Science Foundation on of China under the Grant no. 61365010.

\section{References}

[1] A. Jameson and B. Smyth, "Recommendation to groups," in Adaptive Web, pp. 596-627, 2007.

[2] A. Jameson, "More than the sum of its members: Challenges for group recommender systems," in Proceedings of the Working Conference on Advanced Visual Interfaces, AVI 2004, pp. 48-54, ita, May 2004.

[3] M. Kompan and M. Bielikova, "Group recommendations: Survey and perspectives," Computing and Informatics, vol. 33, no. 2, pp. 446-476, 2014.

[4] L. Quijano-Sanchez, J. A. Recio-Garcia, and B. Diaz-Agudo, "Personality and Social Trust in Group Recommendations," in Proceedings of the 2010 22nd International Conference on Tools with Artificial Intelligence (ICTAI), pp. 121-126, Arras, France, October 2010.

[5] L. Quijano-Sanchez, J. A. Recio-Garcia, B. Diaz-Agudo, and G. Jimenez-Diaz, "Social factors in group recommender systems," ACM Transactions on Intelligent Systems and Technology, vol. 4, no. 1, article no. 8, 2013.

[6] L. Quijano-Sánchez, B. Díaz-Agudo, and J. A. Recio-García, "Development of a group recommender application in a Social Network," Knowledge-Based Systems, vol. 71, pp. 72-85, 2014.

[7] M. Gartrell, X. Xing, Q. Lv et al., "Enhancing group recommendation by incorporating social relationship interactions," in Proceedings of the 16th ACM International Conference on Supporting Group Work, GROUP'10, pp. 97-106, usa, November 2010.

[8] L. Quijanosanchez, J. Reciogarcia, and B. Diazagudo, Group recommendation methods for social network environments, 2011.

[9] I. A. Christensen and S. Schiaffino, "Social influence in group recommender systems," Online Information Review, vol. 38, no. 4, pp. 524-542, 2014. 
[10] F. Supan, K. Takanori, F. Goonnapa et al., "Group modeling: Selecting a sequence of television items to suit a group of viewers," User Modeling and User-Adapted Interaction, vol. 14, no. 1, pp. 37-85, 2004.

[11] F. Ricci, L. Rokach, B. Shapira, and P. B. Kantor, Eds., Recommender Systems Handbook, Springer, 2011.

[12] F. Ortega, J. Bobadilla, A. Hernando, and A. Gutiérrez, "Incorporating group recommendations to recommender systems: Alternatives and performance," Information Processing \& Management, vol. 49, no. 4, pp. 895-901, 2013.

[13] N. A. Najjar and D. C. Wilson, "Differential neighborhood selection in memory-based group recommender systems," in Proceedings of the 27th International Florida Artificial Intelligence Research Society Conference, FLAIRS 2014, pp. 69-74, usa, May 2014.

[14] J. Kelleher and D. Bridge, An Accurate and Scalable Collaborative Recommender, Kluwer Academic Publishers, 2004.

[15] L. Baltrunas, T. Makcinskas, and F. Ricci, "Group recommendations with rank aggregation and collaborative filtering," in Proceedings of the 4th ACM Recommender Systems Conference, RecSys 2010, pp. 119-126, esp, September 2010.

[16] S. Berkovsky and J. Freyne, "Group-based recipe recommendations: analysis of data aggregation strategies," in Proceedings of the 4th ACM Recommender Systems Conference (RecSys '10), pp. 111-118, Barcelona, Spain, September 2010.

[17] G. Linden, B. Smith, and J. York, "Amazon.com recommendations: item-to-item collaborative filtering," IEEE Internet Computing, vol. 7, no. 1, pp. 76-80, 2003.

[18] J. Ben Schafer, F. Dan, J. Herlocker, and S. Sen, Collaborative Filtering Recommender Systems, Springer, Berlin, Germany, 2007.

[19] B. Sarwar, G. Karypis, J. Konstan, and J. Riedl, "Item-based collaborative filtering recommendation algorithms," in Proceedings of the 10th International Conference on World Wide Web (WWW '01), pp. 285-295, 2001.

[20] Y. Zhang, M. Chen, D. Huang, D. Wu, and Y. Li, "IDoctor: personalized and professionalized medical recommendations based on hybrid matrix factorization," Future Generation Computer Systems, vol. 66, pp. 30-35, 2017.

[21] Y. Zhang, "GroRec: a group-centric intelligent recommender system integrating social, mobile and big data technologies," IEEE Transactions on Services Computing, vol. 9, no. 5, pp. 786795, 2016.

[22] Y. Zhang, Z. Tu, and Q. Wang, “TempoRec: Temporal-Topic Based Recommender for Social Network Services," Mobile Networks and Applications, vol. 22, pp. 1182-1191, 2017.

[23] Y. Zhang, D. Zhang, M. M. Hassan, A. Alamri, and L. Peng, "CADRE: Cloud-Assisted Drug REcommendation Service for Online Pharmacies," Mobile Networks and Applications, vol. 20, no. 3, pp. 348-355, 2015.

[24] J. F. McCarthy and T. D. Anagnost, "MUSICFX: an arbiter of group preferences for computer supported collaborative workouts," in Proceedings of the 7th ACM Conference on Computer Supported Cooperative Work (CSCW' '98), pp. 363-372, November 1998.

[25] A. Crossen, J. Budzik, and K. J. Hammond, "Flytrap: Intelligent group music recommendation," in Proceedings of the 2002 International Conference on intelligent User Interfaces (IUI 02), pp. 184-185, usa, January 2002.

[26] C. Baccigalupo and E. Plaza, "A case-based song scheduler for group customised radio," in Proceedings of the International
Conference on Case-Based Reasoning: Case-Based Reasoning Research and Development, pp. 433-448, 2007.

[27] H. Lieberman, N. W. Van Dyke, and A. S. Vivacqua, "Let's Browse: a collaborative web browsing agent," in Proceedings of the 1998 11th Annual ACM Symposium on User Interface Software and Technology, UIST-98, pp. 65-68, November 1998.

[28] K. McCarthy, M. Salamó, L. Coyle, L. McGinty, B. Smyth, and P. Nixon, "CATS: A synchronous approach to collaborative group recommendation," in Proceedings of the FLAIRS 2006 19th International Florida Artificial Intelligence Research Society Conference, pp. 86-91, Melbourne Beach, Florida, USA, May 2006.

[29] L. Ardissono, A. Goy, G. Petrone, M. Segnan, and P. Torasso, "Intrigue: personalized recommendation of tourist attractions for desktop and hand held devices," Applied Artificial Intelligence, vol. 17, no. 8-9, pp. 687-714, 2003.

[30] J. F. Mccarthy, Pocket restaurantfinder: A situated recommender system for groups, 2002.

[31] S. Shin, S.-J. Jang, and S.-P. Lee, “The user-group based recommendation for the diverse multimedia contents in the social network environments," in Proceedings of the 9th International Conference on Dependable, Autonomic and Secure Computing, pp. 202-206, December 2011.

[32] J. K. Kim, H. K. Kim, H. Y. Oh, and Y. U. Ryu, "A group recommendation system for online communities," International Journal of Information Management, vol. 30, no. 3, pp. 212-219, 2010.

[33] J. E. John, “Thomas-kilmann conflict mode instrument," Group \& Organization Management, vol. 1, no. 2, pp. 249-251, 1976.

[34] J. S. Dyer and R. K. Sarin, "Group preference aggregation rules based on strength of preference," Management Science, vol. 25, no. 9, pp. 822-832 (1980), 1979.

[35] G. Guo, J. Zhang, and N. Yorke-Smith, "A novel bayesian similarity measure for recommender systems," in Proceedings of the 23rd International Joint Conference on Artificial Intelligence, IJCAI 2013, pp. 2619-2625, chn, August 2013. 


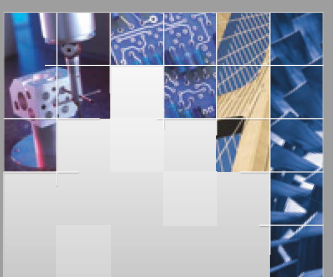

\section{Enfincering}
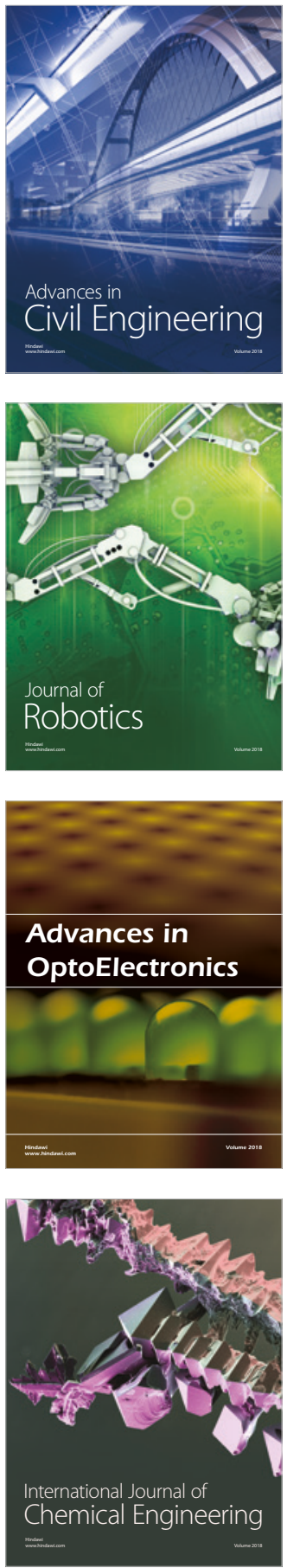

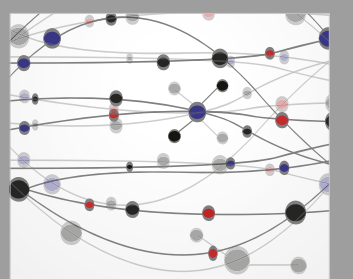

\section{Rotating \\ Machinery}

The Scientific World Journal

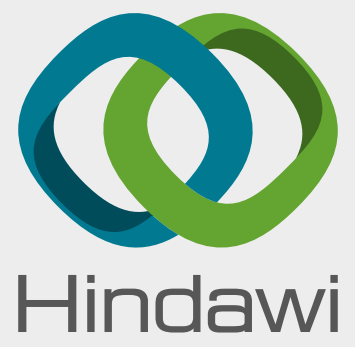

Submit your manuscripts at

www.hindawi.com
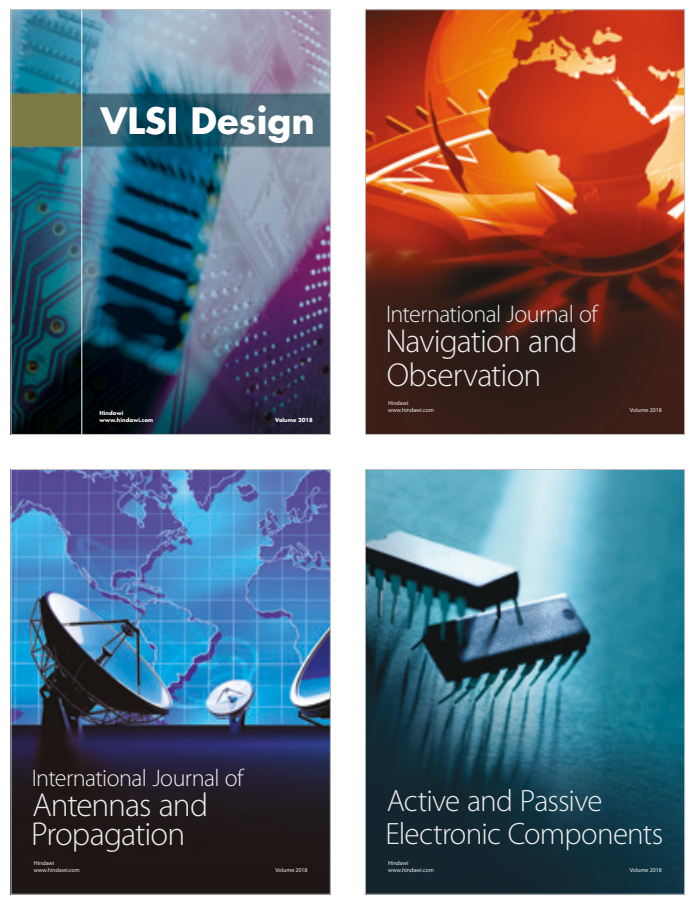
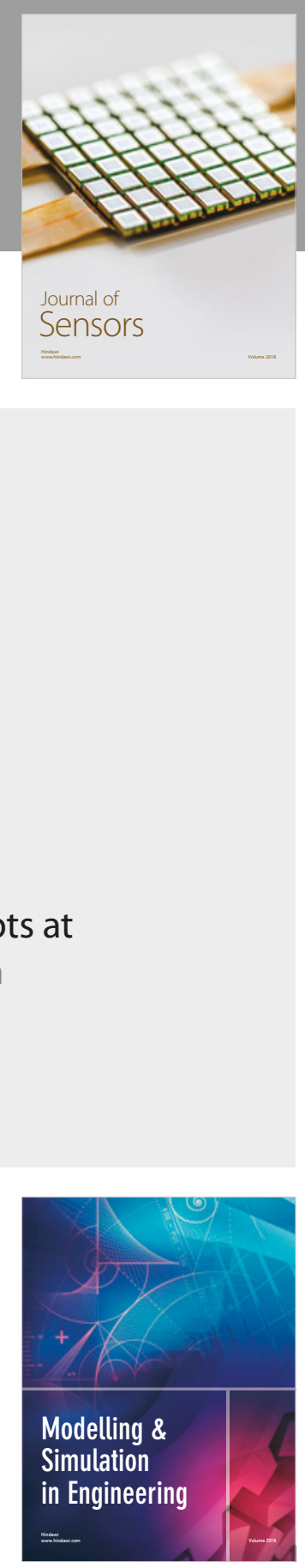

\section{Advances \\ Multimedia}
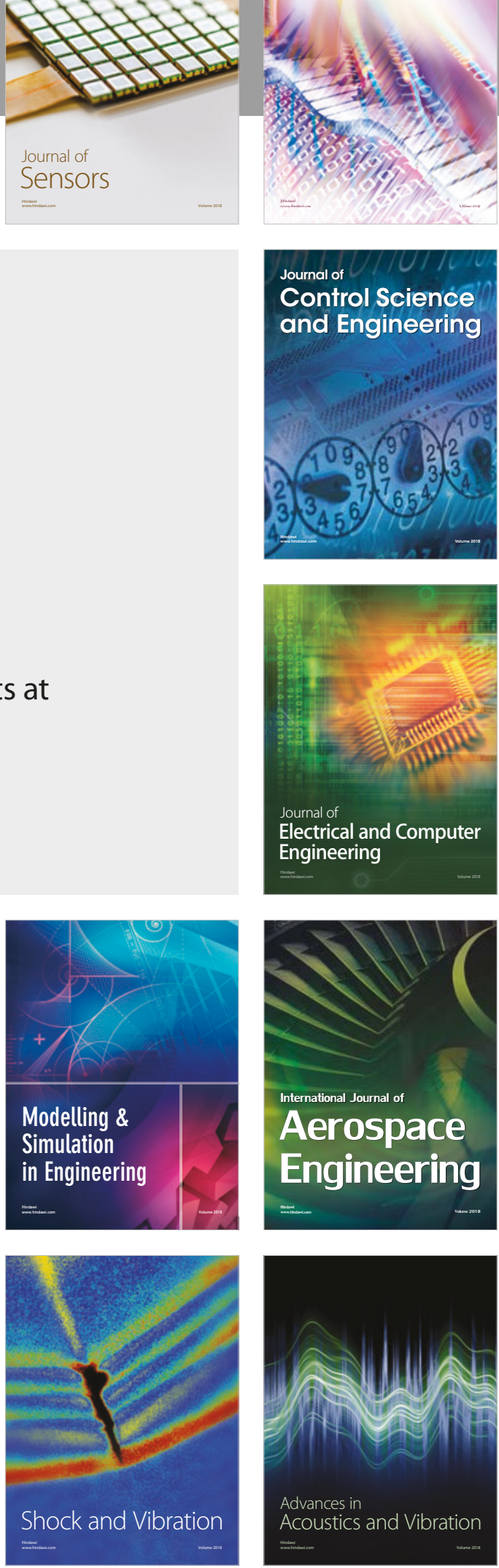